\title{
Pulsed Fibre Laser with Spatial and Temporal Control
}

\author{
Paulo Almeida*a, Philip Gorman**a , Jaclyn S. Chan ${ }^{\mathrm{b}}$, Natasha Vukovic ${ }^{\mathrm{b}}$, Christophe A. Codemard ${ }^{\mathrm{a}}$, and \\ Michalis N. Zervas ${ }^{a, b}$ \\ aAdvanced Laser Laboratory, SPI Lasers UK Ltd, Southampton SO30 2QU, UK; \\ ${ }^{\mathrm{b}}$ Optoelectronics Research Centre, University of Southampton, Southampton SO17 1BJ, UK
}

\begin{abstract}
We report on novel approach to switch the optical beam profile in a fibre laser using a short piece of special passive fibre. The switching mechanism relies on selective wavelength resonance between the core and a high index surrounding ring in the fibre. Beam switching at $530 \mathrm{kHz}$ from a Gaussian beam shape to a flat-top beam with a ring with corresponding $\mathrm{M}^{2}$ from 1.4 to 3.5 is demonstrated in a modified industrial pulsed fibre laser.
\end{abstract}

Keywords: High power fiber laser, beam delivery, beam switching

\section{INTRODUCTION}

Pulsed fibre lasers are now de-facto laser of choice in a number of industrial applications, such as micromachining, drilling and marking thanks to their flexibility, performance and cost. The most agile pulsed fibre lasers use a MOPA (MasterOscillator Power-Amplifier) architecture typically based on semiconductor laser diode source and doped-fibre amplification stages [1]. This approach allows for temporal control with ability to select waveforms, pulse repetition-rate and energy to optimize the desired application.

In this paper, we present a novel approach that enables full spatio-temporal control in a pulsed fibre laser with fast, electronically controllable switching of the output beam. Current approaches in pulsed MOPA Fibre Lasers typically rely on mechanical mode coupling [2] that is inherently slow or by using spatial-light modulators [3-4] that are power limited and require feedback loop control. Here, a redENERGY G4 pulsed fibre laser from SPI Lasers Ltd [5] is modified to accommodate two semiconductor seed diodes operating at different wavelengths, while a piece of resonant fibre is incorporated in the beam delivery fibre of the MOPA. The resonant fibre operates in similar fashion as a WDM coupler where different wavelengths propagate in different waveguides thus allowing fast switching of the beam profile.

\section{PRINCIPLE OF OPERATION}

The principle of operation of the fibre mode-coupler relies on the wavelength dependence of the field delocalization from the core into the ring at resonant wavelength [6], as illustrated in Figure 1, similar to a fibre fused coupler.
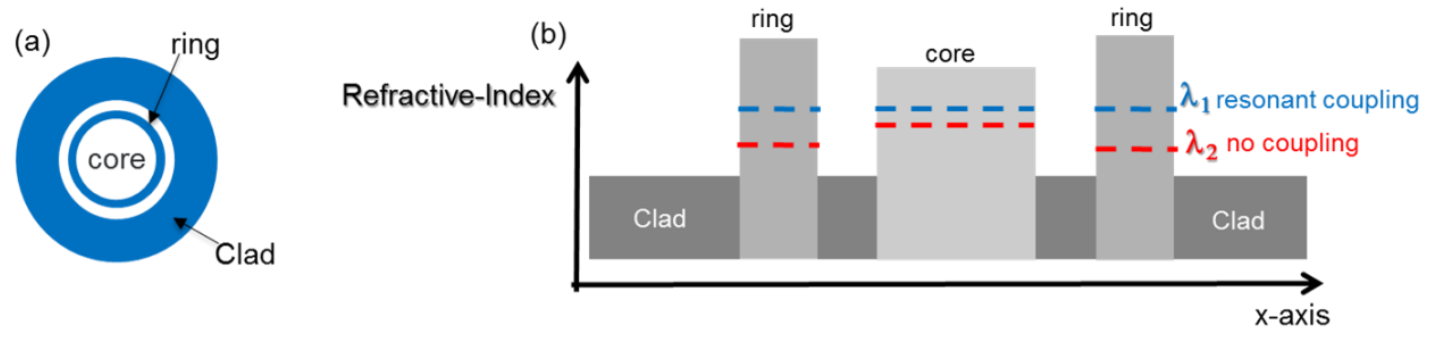

(c)

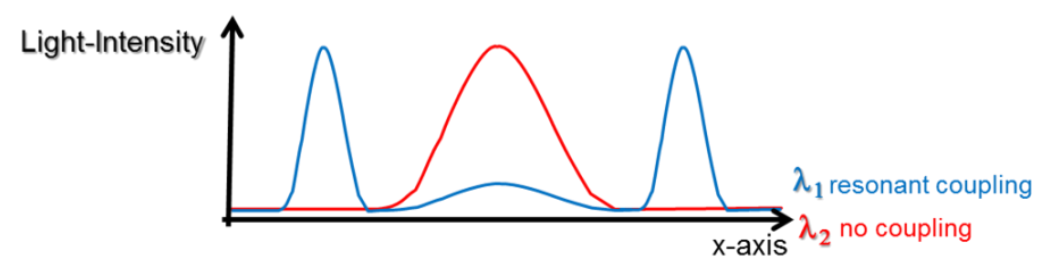

Figure 1. Illustration of operating principle of fibre mode converter: (a) fibre cross-section, (b) refractive-index profile, (c) beam profile conversion due to resonant coupling between core and ring modes.

*paulo.almeida@spilasers.com; phone: +44(0)2380597230

**Dr. Philip Gorman is now with QinetiQ Ltd. 
At resonant wavelength, the core fundamental mode propagation constant matches that of one of the modes in the ring, and full power transfer is possible between the core and ring mode. The fibre coupler length for full power transfer depends on the field overlap between the two modes. A weak field overlap requires a longer coupler length to fully transfer the power between the two modes. At non resonant wavelength, the power transfer between modes can be small, and therefore the beat length is not important to be considered.

A resonant fibre was manufactured using MCVD method, and comprises silica core surrounded by high-index rings. The fibre core diameter was $20 \mu \mathrm{m}$, core NA of 0.06 , and ring of $2.5 \mu \mathrm{m}$ thickness is $\sim 6 \mu \mathrm{m}$ apart from the core. These parameters suggest that such fibre is well suited for high-energy pulse delivery.

The 2D refractive-index of the fibre was measured using an Interfiber Analysis IFA-100 analyzer. We used a full vectorial modal solver based on finite element method to numerically calculate the effective indices of the core and ring modes, taking into account the measured refractive-index profile. The calculation results showed that the resonant wavelength for the fabricated fibre was near $1067 \mathrm{~nm}$, which was also confirmed by directly measuring the near-field beam profile at different wavelengths. In the wavelength region between $1045 \mathrm{~nm}$ and $1055 \mathrm{~nm}$, there was negligible coupling from the core to the ring, whilst strong resonant coupling from the core to the first ring was observed in the wavelength region between $1060 \mathrm{~nm}$ and $1067 \mathrm{~nm}$.

The principle of operation of the beam switchable pulsed fibre laser is based on a modified MOPA configuration, comprising a directly modulated low power semiconductor seed and a chain of fibre amplifiers, as shown in Figure 2 . The delivery fibre is modified to include a short section of the resonant core-ring fibre, and the MOPA operating wavelength can be controlled by switching between two seed diodes with different emission wavelength. One seed diode is made to operate at a wavelength near resonance, and the other seed at a non-resonant wavelength. The wavelength of the two seeds is also chosen so that the amplifier gain is substantially identical for the two seeds.

\section{EXPERIMENTAL CHARACTERIZATION}

The experimental setup used to achieve beam switchable pulsed fibre laser MOPA is shown in Figure 2. It includes a G4 laser [5], which is modified to drive two pulsed laser diodes operating at different wavelengths of $1053 \mathrm{~nm}$ and $1067 \mathrm{~nm}$, that are combined together with a fibre coupler and amplified in a MOPA using GTWave ${ }^{\circledR}$ Ytterbium-doped fibre amplifiers. The output of the MOPA delivery fibre is spliced to a $25 \mathrm{~cm}$ long piece of the resonant fibre. The two diodes are externally triggered by a pulse generator operating at the selected clock frequency of $530 \mathrm{kHz}$, while a second pulse generator is used to alternatelly switch on and off on-the-fly between the two seed diodes. The capability of switching on-demand between the two wavelengths using the G4 electronic interface was first directly tested with a CCD beam imaging camera. However, the test was limited to observing switching speeds of a few $\mathrm{Hz}$ by the camera maximum frame rate of $<10 \mathrm{~Hz}$.

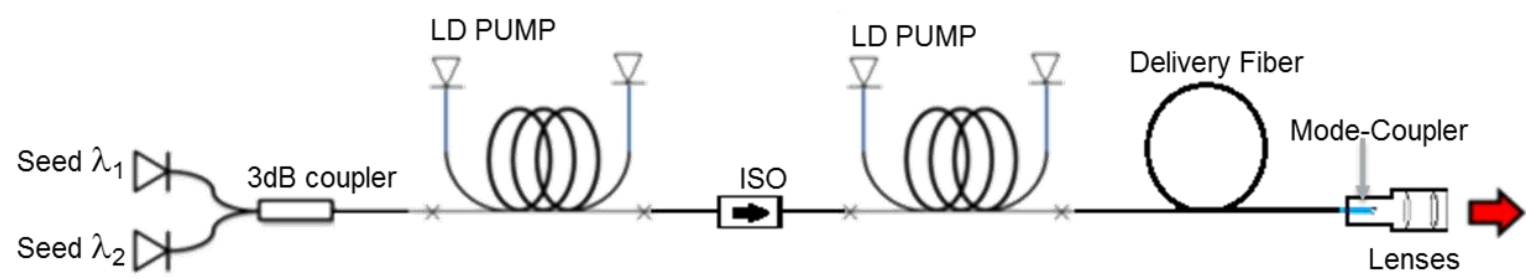

Figure 2. Experimental setup of beam switchable pulsed fibre laser MOPA.

Higher speed measurements were made using the setup illustrated in Figure 3. The output of the laser was passed through a bulk diffraction grating, which spatially separates the two operating wavelengths. This produced two separate beams that were directed to separate high-speed photodetectors, and individually coupled into a digital sampling oscilloscope. It should be noted that this setup tests the capability of the electronic interface to switch on-demand between two seeds when externally triggered, as the switching of beam profiles occurs as in any fibre coupler at the speed-of-light in the fibre medium. 


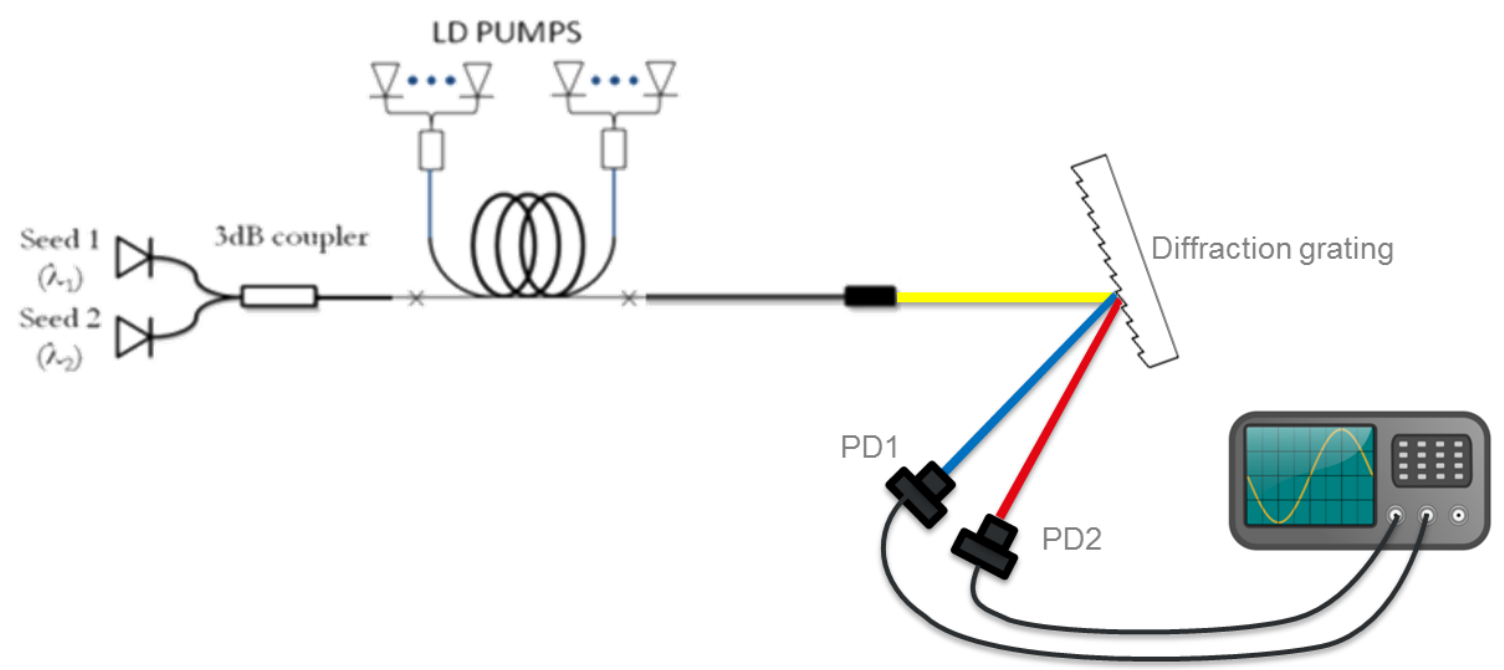

Figure 3. Setup Schematic for testing high-speed beam switching.

Figure 4 shows the pulse trains when the second pulse generator was set to operate at the frequencies of $33 \mathrm{kHz}$, which produced a burst of $530 \mathrm{kHz}$ pulses at each wavelength, and $256 \mathrm{kHz}$, which produces pulse to pulse switching. The switching speed is twice the frequency of the second signal generator, as the signal from the second generator switches seed $\lambda_{1}$ when is high, and seed $\lambda_{2}$ when is low. The output beam profile and $\mathrm{M}^{2}$ beam quality were measured with a OPHIR Spiricon M200 when the laser operates in the two different wavelengths. Figure 4 also shows the measured beam profiles when the laser is operated at $1053 \mathrm{~nm}$ with nearly diffraction-limited beam quality with $\mathrm{M}^{2}$ of 1.4 , and when the laser is operated at $1067 \mathrm{~nm}$ with nearly flat-top beam in the core and light in the ring with $\mathrm{M}^{2}$ of 3.5.
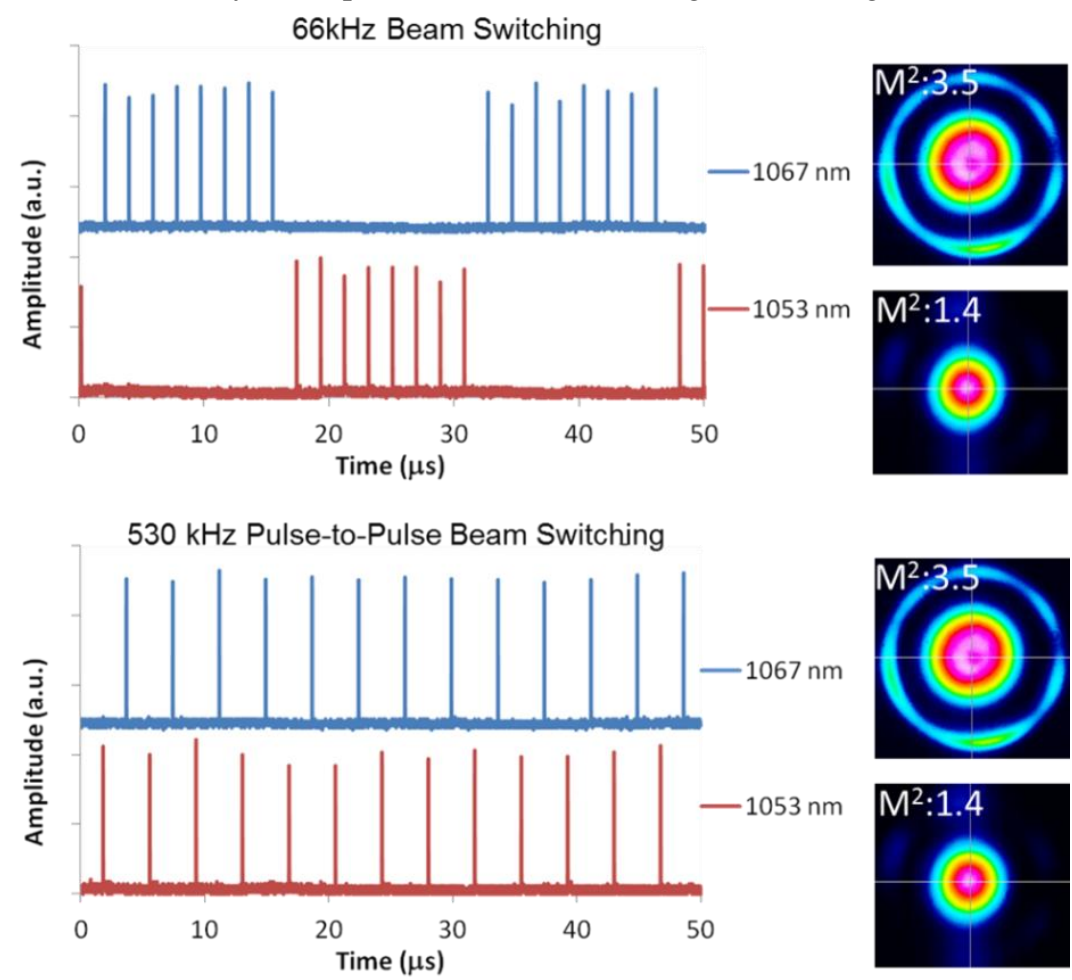

Figure 4. Measured pulse trains at the wavelengths of $1067 \mathrm{~nm}$ and $1053 \mathrm{~nm}$ with on-the-fly fast switching, and corresponding measured beam profile and $\mathrm{M} 2$ beam quality. 


\section{CONCLUSION}

In conclusion, we demonstrate fast electronically controlled output mode switching from an all-fibre pulsed fibre laser using a dual wavelength seed configuration in the G4 electronic interface ${ }^{\circledR}$ developed for industrial integration. Switching speeds up to $530 \mathrm{kHz}$ were experimentally demonstrated, limited by the selected clock frequency for pulse triggering. This proposed architecture can be operated at higher switching speeds, mostly limited by the maximum pulse repetition frequency allowed by the laser. The mode switching occurs in a $25 \mathrm{~cm}$ short section of specialty fibre spliced to the output of the delivery fibre where light from the core couples into a surrounding high-index ring when operated at resonant wavelength. The light in the core is unaffected when operating at non resonant wavelength. Complete power transfer from the core to the ring at resonant wavelength was not observed in our experiments. Initial investigations revealed that this is due to non-uniformity of the refractive-index profile of the ring, which causes unwanted coupling between different modes in the ring. Other fibre fabrication methods than MCVD are being considered for better uniformity of the refractive-index of the ring, such as rod-intube.

\section{REFERENCES}

[1] Zervas MN, Durkin M, Ghiringhelli F, et al., "High peak power, high rep-rate pulsed fibre laser for marking applications", Proceedings of The Society Of Photo-Optical Instrumentation Engineers (SPIE) Vol. 6102 p. Q1020 (2006).

[2] Liu, T., Chen, S., Qi, X., and Hou J., "High-power transverse-mode-switchable all-fiber picosecond MOPA," Opt. Express 24(24), 27821-27827 (2016).

[3] R. Beck, J. Parry, W. MacPherson, A. Waddie, N. Weston, J. Shephard, and D. Hand, "Application of cooled spatial light modulator for high power nanosecond laser micromachining," Opt. Express 18, 17059-17065 (2010).

[4] B. M. Zhang, Y. Feng, D. Lin, J. Price, S. Alam, J. Nilsson, P. Shum, D. Payne, and D. Richardson, "Radially polarised Yb-fiber MOPA producing $10 \mathrm{~W}$ output using SLM based pulse shaping for efficient generation of arbitrary shaped picosecond pulses," in Lasers Congress 2016 (ASSL, LSC, LAC), OSA Technical Digest (online) (Optical Society of America, 2016), paper ATh3A.3.

[5] http://www.spilasers.com/industrial-fiber-lasers/redenergy-g4/

[6] A. C. Boucouvalas, "Coaxial optical fiber coupling," in Journal of Lightwave Technology, vol. 3, no. 5, pp. 1151-1158, Oct 1985 . 